04

\title{
Снижение донного сопротивления и создание подъемной силы с использованием микроволнового разряда в сверхзвуковом потоке
}

\author{
() В.Л. Бычков, ${ }^{1}$ Л.П. Грачев, ${ }^{2}$ И.И. Есаков, ${ }^{3}$ А.В. Семенов ${ }^{3}$ \\ ${ }^{1}$ Московский государственный университет им. М.В. Ломоносова, \\ 119991 Москва, Россия \\ ${ }^{2} \mathrm{AO}$ „Московский радиотехнический институт РАН“, \\ 117519 Москва, Россия \\ ${ }^{3}$ Московский университет им. С.Ю. Витте, \\ 115432 Москва, Россия \\ e-mail: iesakov@yandex.ru
}

Поступило в Редакцию 14 декабря 2019 г.

В окончательной редакции 13 февраля 2020 г.

Принято к публикации 13 февраля 2020 г.

Представлены результаты экспериментальных исследований силового воздействия глубоко подкритического микроволнового разряда, зажженного в квазиоптическом электромагнитном пучке, на тела, помещенные в сверхзвуковой поток воздуха.

Ключевые слова: аэродинамика, микроволновый разряд, инициатор разряда, силовое воздействие.

DOI: $10.21883 /$ JTF.2020.08.49537.407-19

\section{Введение}

Гиперзвуковой полет является очень сложным процессом. Его исследования продолжаются уже более 50 лет. Скейлинговые законы для необходимой энергии и тепловых нагрузок являются нелинейными относительно числа Маха (М). Аэродинамическое сопротивление увеличивается примерно как $\rho \mathrm{M}^{2}$, где $\rho$ является плотностью газа, а аэродинамический нагрев увеличивается примерно как $\rho \mathrm{M}^{3}$. Плотность атмосферы уменьшается с высотой примерно в $10^{3}$ на каждых $50 \mathrm{~km}$ по высоте. Ограничения по тепловым режимам говорят о том, что гиперзвуковой полет на числах Маха больше 10 может быть достигнут только на очень больших высотах

Для того чтобы уменьшить лобовое сопротивление, аэродинамический профиль высокоскоростного аппарата имеет вид остроугольного конуса; однако эта геометрия делает трудным активное охлаждение аппарата. Прогресс в конструировании летательных аппаратов в начале 1990-х годов потребовал изучения возможности применения других методов снижения аэродинамического сопротивления.

Для снижения сопротивления при сверхзвуковых скоростях известны два основных физических эффекта при подаче дополнительного тепла в поле течения. Первый эффект заключается в снижении плотности в передней части обтекаемого тела (из-за повышения температуры), это предполагает использование постоянного подогрева. Другой механизм представляет собой объединение эффектов низкой плотности в следе за зоной нагрева и поля течения вокруг тела, это может привести к созданию совершенно иного поля течения. Величина влияния этого эффекта на поле течения отличается для различных аэродинамических форм обтекаемого тела.
Этот эффект является очень сильным в случае тупых тел, и заключается в изменении течения около тупого тела на течение, более похожее на течение около конического тела. Эффективность эффекта значительно возрастает с увеличением числа Маха.

Реализация изменений параметров внешнего потока и уменьшения аэродинамического сопротивления с помощью выделения дополнительного тепла в потоке возможна с использованием плазмохимических и химикотермических механизмов. Были предложены схемы модификации и управления потоками у поверхности гиперзвуковых летательных аппаратов. Эти схемы включали новые подходы к генерации плазмы, МГД-управлению потоками и производству электроэнергии, созданию встречных потоков горячих газов и другим чисто тепловым методам.

Внедрение плазмы в высокоскоростные аэродинамические поля течений с присущими им сильными градиентами физических параметров приводит к рождению множества физических процессов, включая сложные взаимодействия, происходящие при прохождении ударной волны (УВ).

Создание плазмы осуществляется с помощью электрических разрядов различного типа. Стримерные (нитевидные) разряды были исследованы в целях поиска методов снижения аэродинамического сопротивления при сверхзвуковых и гиперзвуковых скоростях. Эти разряды могут быть очень неоднородными и в пространстве, и во времени. Они создаются с помощью высокочастотных разрядов и СВЧ (микроволновых) разрядов или при инжекции электронных пучков через небольшие отверстия.

Высокочастотные импульсные разряды были исследованы в работах [1-4]. Модели как с хорошей аэродинами- 
ческой формой, так и конуса-цилиндра были испытаны при числе Маха 2. Модели имели острые электроды в голове модели для того, чтобы сконцентрировать действие электрического поля. В работах использовался источник электропитания с несущей частотой $13-16 \mathrm{MHz}$ и амплитудой модуляции $100 \mathrm{~Hz}$. Наибольшее внимание исследователей с точки зрения эффективности воздействия на потоки привлекает СВЧ разряд. Это связано прежде всего с его высокой энергетической эффективностью. Практически вся энергия источника СВЧ потребляется таким разрядом при давлениях выше 30 Torr.

Результаты исследований воздействия СВЧ разряда на газодинамические процессы представлены в работах $[5-20]$.

\section{Эксперименты с микроволновым подкритическим разрядом в высокоскоростном потоке воздуха}

Настоящая работа посвящена экспериментальному исследованию взаимодействия микроволнового подкритического разряда с высокоскоростным потоком воздуха.

Схема реализации глубоко подкритического микроволнового разряда в сверхзвуковом потоке приведена на рис. 1. На нем даны также характерные параметры используемой в экспериментах установки.

В установке в течение времени $\pi_{f l} \approx 1 \mathrm{~s}$ создается затопленная осесимметричная струя воздуха с начальным диаметром $30 \mathrm{~mm}$, числом Маха потока $\mathrm{M}=2$, скоростью воздуха $v \approx 500 \mathrm{~m} / \mathrm{s}$, статическом давлении $p_{\text {stat }} \approx 100$ Torr, статической температуре $T_{\text {stat }} \approx 150 \mathrm{~K}$, давлением и температурой торможения $p_{\text {stag }} \approx 550$ Torr, $T_{\text {stag }} \approx 300 \mathrm{~K}$ соответственно. В экспериментах используется электромагнитное излучение с длиной волны $\lambda=12.5 \mathrm{~cm}$. Излучение сформировано в квазиоптический линейно поляризованный пучок с характерным поперечным размером $9 \times 9 \mathrm{~cm}$ при ампли-

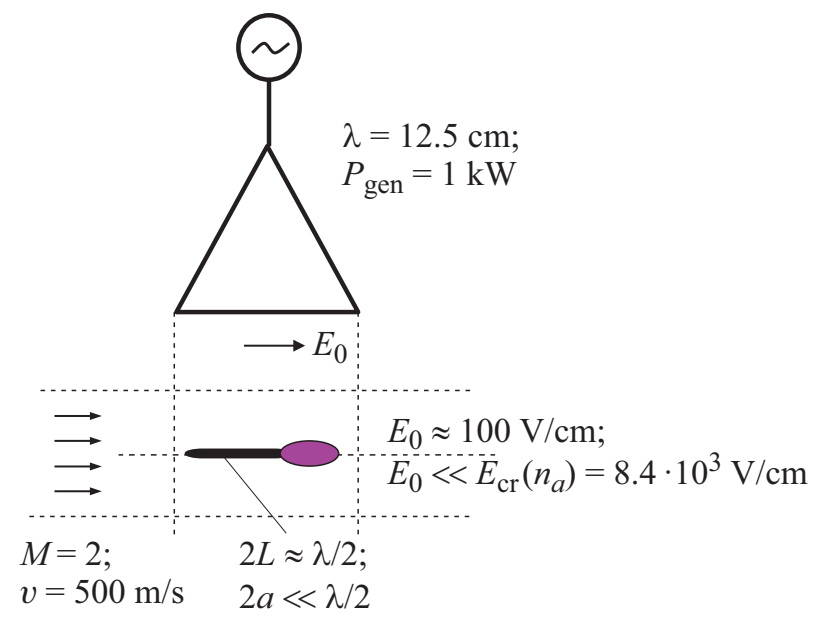

Рис. 1. Схема реализации микроволнового разряда в сверхзвуковом потоке.

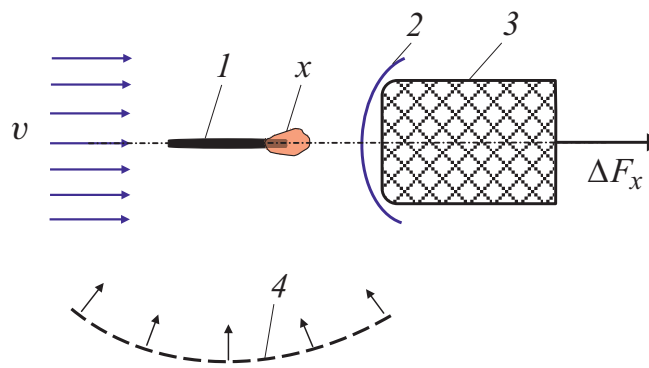

$a$

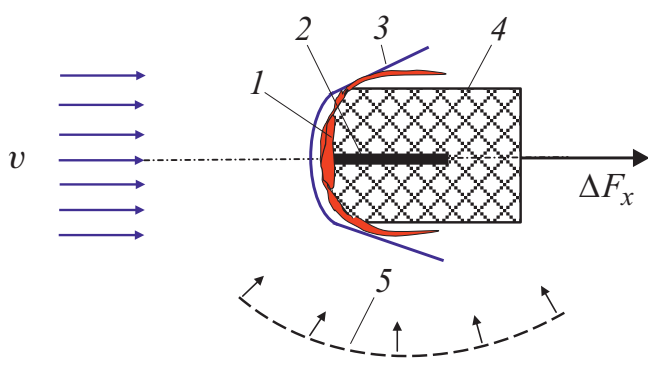

$b$

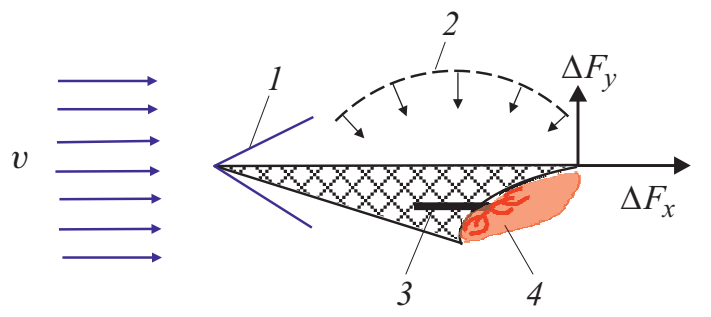

$c$

Рис. 2. Схема эксперимента с зажиганием глубоко подкритического инициированного микроволнового разряда: $a-$ перед головной УВ: 1 - инициатор, 2 - УВ, 3 - модель, 4 - микроволновое излучение; $b-$ за головной УВ: $1-$ разряд, 2 - инициатор, 3 - УВ, 4 - модель, 5 - микроволновое излучение; $c-$ в кормовой части модели: $1-\mathrm{УВ,}$ 2 - микроволновое излучение, 3 - инициатор, $4-$ разряд.

туде его электрической компоненты в рабочей области $E_{0} \approx 100 \mathrm{~V} / \mathrm{cm}$. Это поле существенно меньше минимального, критического электрического поля, при котором происходит пробой воздуха в воздушной струе при экспериментальной его плотности: $E_{0} \ll E_{\mathrm{cr}}=8.4 \cdot 10^{3} \mathrm{~V} / \mathrm{cm}$.

Для реализации микроволнового разряда в поле с такой глубокой подкритичностью $\xi=E_{\mathrm{cr}} / E_{0}=84$ он инициировался металлическим электромагнитным вибратором в виде цилиндра длиной $2 L \approx \lambda / 2$ и диаметром $2 a \ll \lambda / 2$. Разряд такого типа по принятой терминологии называется глубоко подкритическим разрядом.

Ранее были опубликованы результаты экспериментов, схемы которых приведены на рис. 2 [2,21]. В них измерялись и сравнивались продольные аэродинамические силы $F_{x}$, действующие на помещенные в сверхзвуковой поток тела простейших форм при отсутствии и наличии микроволнового разряда. При этом разряд зажигался перед головной УВ модели, между головной УВ и по- 

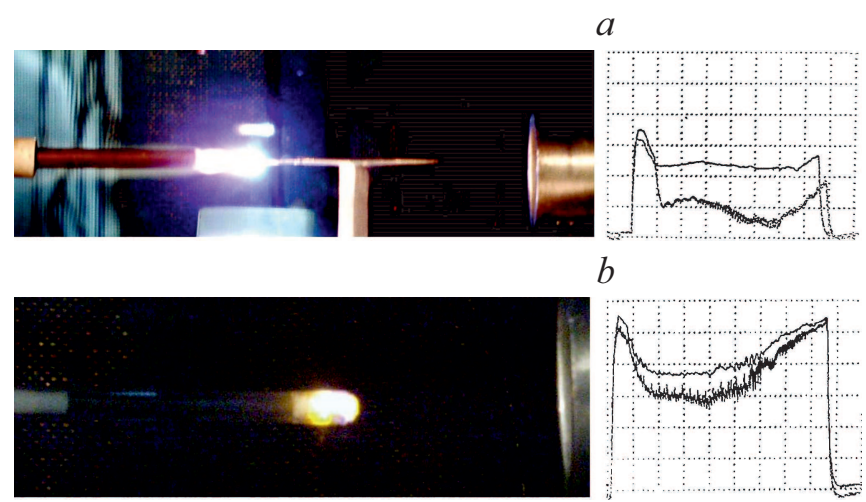

$b$
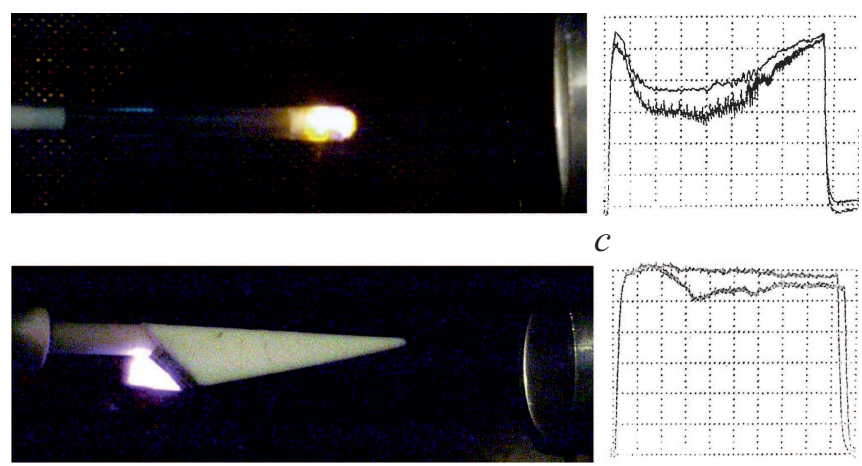

Рис. 3. Фотографии разряда при его зажигании: $a-$ перед головной УВ, $b-$ за головной УВ, $c-$ в кормовой части модели и соответствующие им осциллограммы измеряемых $F_{x}$.
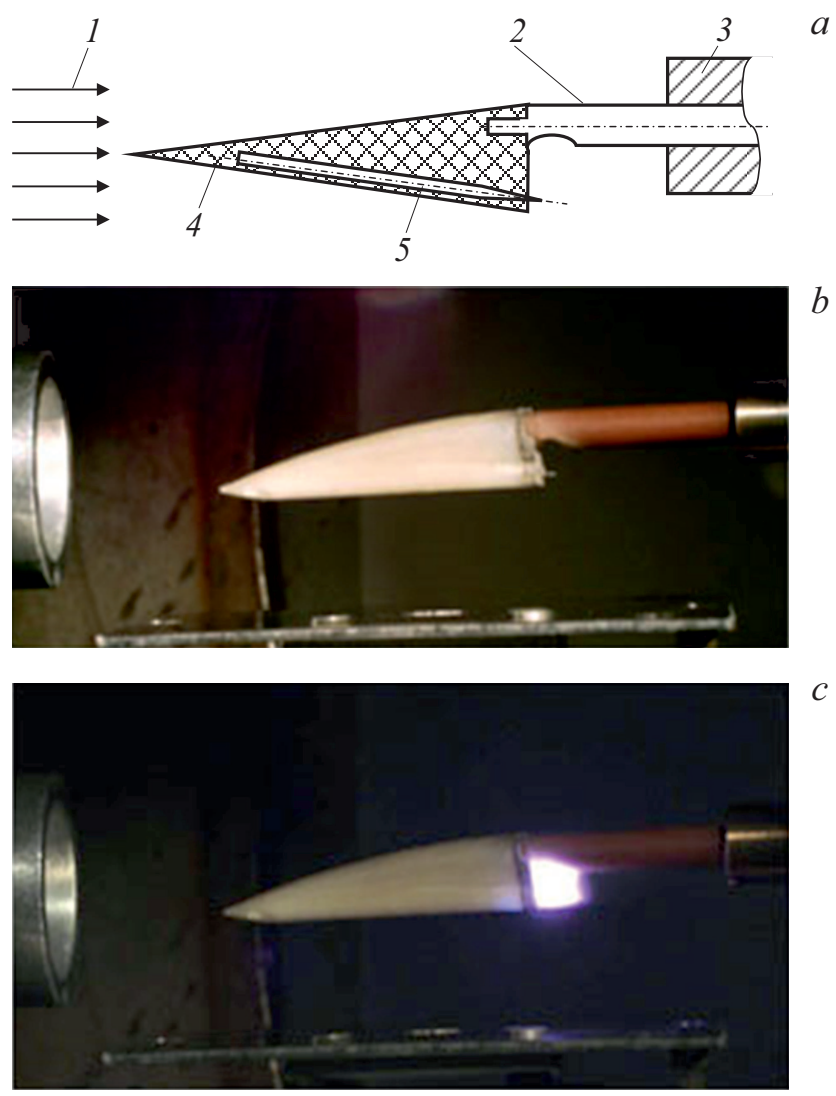

Рис. 4. Эксперимент по изучению воздействия глубоко подкритического микроволнового разряда на донное сопротивление модели, помещенной в сверхзвуковой поток: $a-$ схема эксперимента: 1 - сверхзвуковой поток, $2-$ крепление модели, 3 - аэродинамические весы, 4 - модель, 5 - инициатор; $b-$ фотография модели в потоке, $c-$ фотография модели с горящим разрядом.

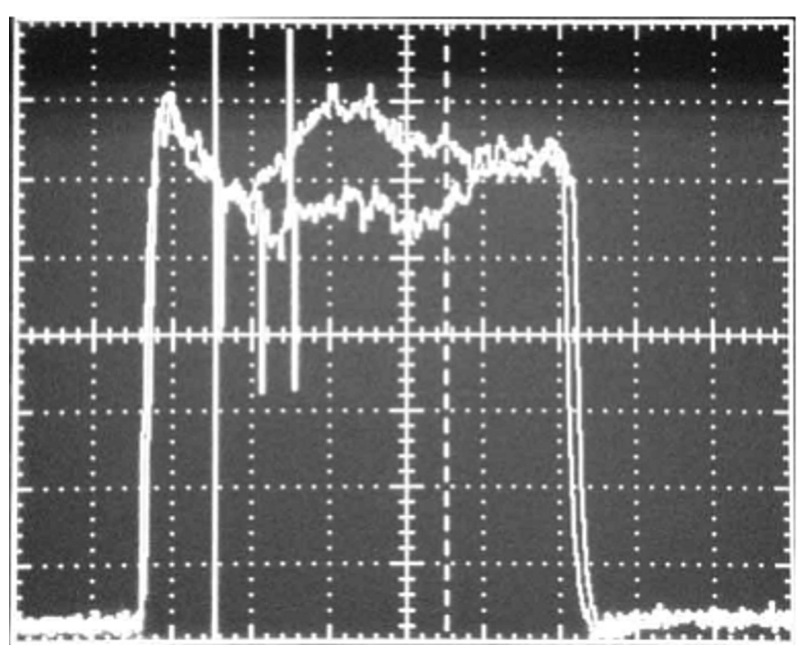

Рис. 5. Типичные осциллограммы Fx в эксперименте по изучению воздействия глубоко подкритического микроволнового разряда на донное сопротивление модели.
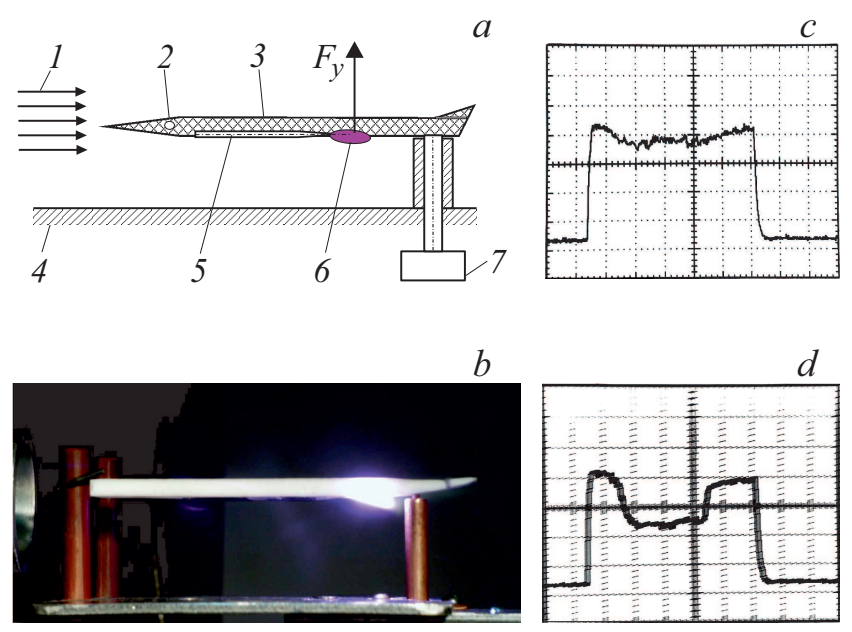

Рис. 6. Эксперимент по созданию боковой силы $F_{y}$ с помощью микроволнового разряда, созданного на поверхности модели, противоположной падающему микроволновому излучению: $a$ - схема эксперимента: 1 - поток, 2 - ось, 3 - модель, 4 - экран, 5 - инициатор, $6-$ разряд, $7-$ измеритель; $b$ - фотография модели с горящим разрядом; $c$ и $d-$ осциллограммы измеряемой $F_{y}$ без разряда и с разрядом.

верхностью тела и в его кормовой области с наклонной относительно направления потока поверхностью.

На рис. 3 приведены соответствующие фотографии (на фотографиях направление потока справа налево) и осциллограммы измеряемых $F_{x}$. На осциллограммах верхние кривые соответствуют отсутствию разряда, а нижние - наличию.

В условиях этих экспериментов при зажигании разряда перед головной УВ уменьшение $F_{x}$ достигало $70-80 \%$; при его горении за головной УВ - до 20\%; а при зажигании разряда в кормовой области модели возникающая движущая, продольная плазменно- аэроди- 

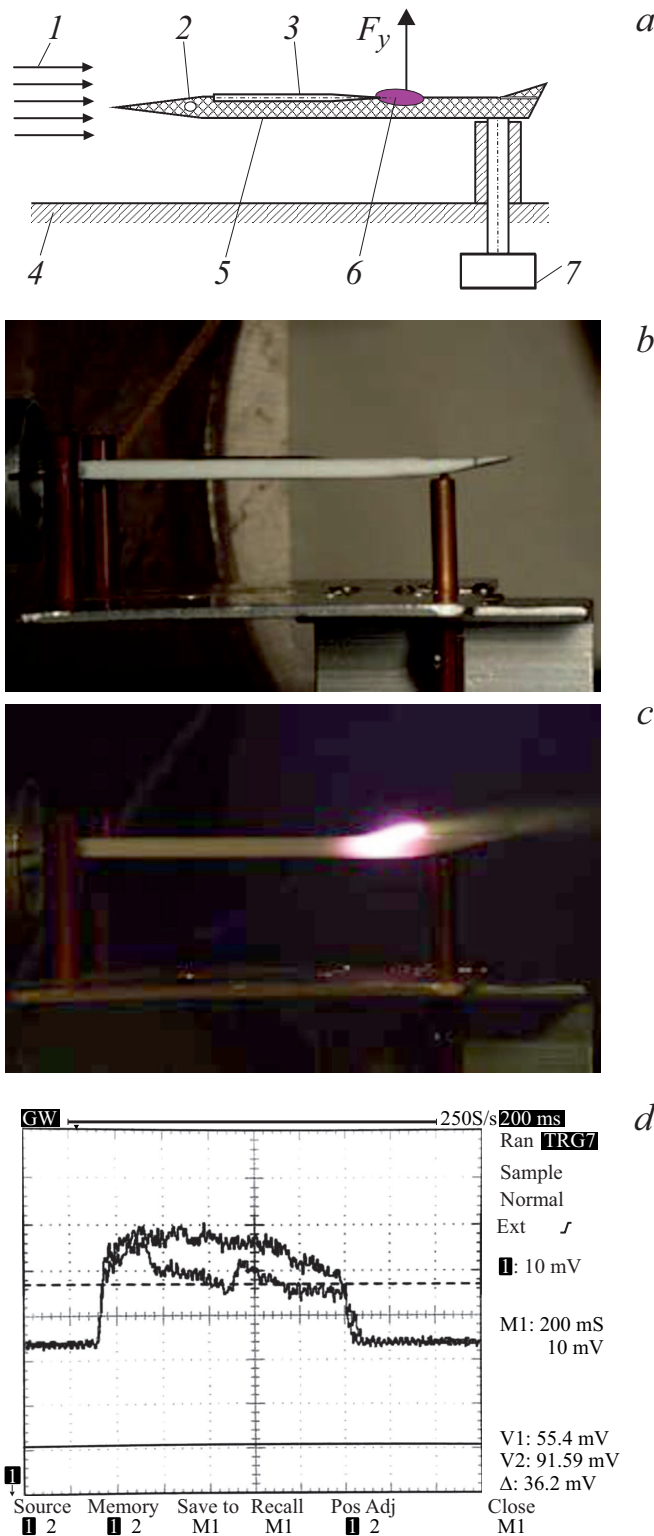

Рис. 7. Эксперимент по созданию боковой силы $F_{y}$ с помощью микроволнового разряда, созданного на поверхности модели, обращенной к микроволновому излучению: $a-$ схема эксперимента: 1 - сверхзвуковой поток, $2-$ ось, $3-$ инициатор, 4 - экран, 5 - модель, 6 - разряд, 7 - измеритель $F_{y}$; $b-$ фотография модели, $c-$ фотография модели с горящим разрядом; $d-$ осциллограмма измеряемой $F_{y}$ без разряда и с разрядом.

намическая сила достигала $20 \%$ от начальной $F_{x}$. При этом в экспериментах реализовывался микроволновый разряд при мощности электромагнитного пучка почти в $10^{4}$ раз меньшей, чем его мощность, необходимая для обеспечения критического пробойного уровня поля.

Столь убедительные результаты сделали обоснованным продолжение данных исследований и, прежде всего, в направлениях, имеющих определенную перспективу в практике. Были выполнены опыты по воздействию на донное сопротивление модели и по реализации с его помощью „подъемной“ силы.

На рис. 4 приведена схема эксперимента по изучению воздействия глубоко подкритического микроволнового разряда на донное сопротивление модели, помещенной в сверхзвуковой поток, фотография модели в потоке и ее фотография с горящим разрядом.

На рис. 5 приведены наложенные друг на друга две характерные осциллограммы измеряемых $F_{x}$ : при отсутствии разряда - верхняя кривая и его наличии нижняя. Из них следует, что при горении разряда уменьшение $F_{x}$ достигало $19 \%$. Эта величина по масштабу соизмерима с типичной величиной донного сопротивления тел со „срезанной“ кормой по отношению к действующей на них полной силе $F_{x}$. Таким образом, реализация в кормовой области модели разряда практически полностью ликвидировала силу ее донного сопротивления.

На рис. 6 приведена схема эксперимента по созданию подъемной силы $F_{y}$ с помощью микроволнового разряда, зажженного на противоположной микроволновому излучателю поверхности модели. На нем же дана фотография модели с горящим разрядом и осциллограммы измеряемой $F_{y}$ без разряда и с разрядом. Хвостовая часть модели имеет наклон поперек потока, который обеспечивал ее

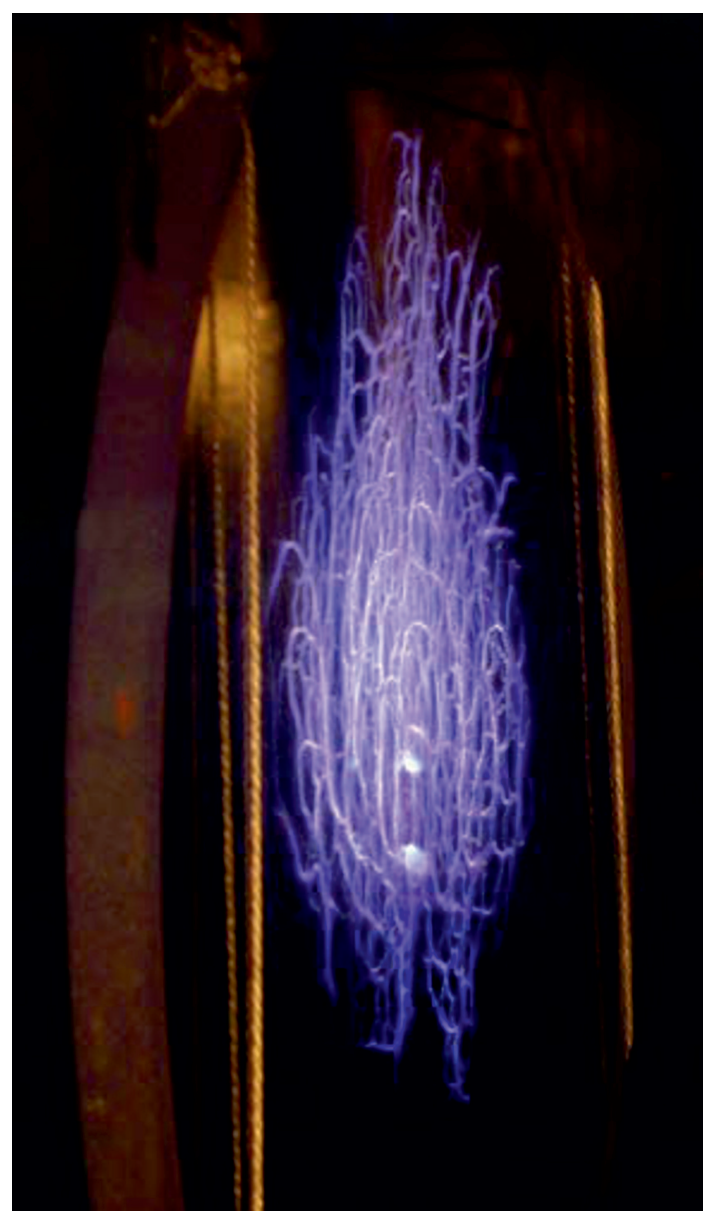

Рис. 8. Микроволновый разряд в подкритической поверхностно развитой форме. 
стабильность в потоке и наличие на весах исходной силы $F_{y} \approx 35$ gf. Из осциллограмм следует, что при горении разряда она уменьшалась на $10 \mathrm{gf}$.

Таким образом, эксперимент продемонстрировал принципиальную возможность реализации управляющего силового плазменно-газодинамического воздействия с помощью микроволнового разряда.

Аналогичный эксперимент был выполнен и с зажиганием разряда на обращенной к микроволновому излучателю поверхности модели. Его схема приведена на рис. 7. На нем же приведены фотографии помещенной в поток модели без разряда и с горящим на ее поверхности разрядом, а также характерные соответствующие им осциллограммы измеряемой $F_{y}$, которые подтверждают и в этом случае возможность воздействия на $F_{y}$.

Описанные эксперименты проводились с глубоко подкритическим привязанным к инициатору микроволновым разрядом. В этом случае силовое плазменногазодинамическое воздействие на поверхность тела носит сравнительно локальный характер.

В то же время микроволновый разряд может быть реализован и в подкритической поверхностно развитой форме. Пример такого разряда на кварцевой пластине приведен на рис. 8. Видно, что в этом случае разряд существует на значительной поверхности. Как показали исследования [22-24], с помощью такого разряда можно эффективно воздействовать на пограничный слой при обтекании тел высокоскоростным потоком. Но, кроме того, можно рассчитывать на значительное увеличение силового воздействия.

\section{Заключение}

В работе представлены результаты экспериментальных исследований воздействия глубоко подкритического микроволнового разряда, зажженного в квазиоптическом электромагнитном пучке на параметры обтекания моделей, помещенных в сверхзвуковой поток воздуха.

Необходимо отметить, что в присутствии плазмы микроволнового разряда исследователями зафиксировано значительное (более чем на два порядка) увеличение скорости горения топливной смеси [25]. В связи с этим становится возможным создавать большие управляющие воздействия, впрыскивая горючую смесь в область поверхностного микроволнового разряда. Таким образом, можно обеспечить уже не локальную, а распределенную по поверхности силу требуемой в практике величины. Таким воздействием можно оперативно управлять по времени, включая и выключая разряд по требуемой программе. Именно в этом направлении и могут быть предприняты дальнейшие экспериментальные исследования.

\section{Конфликт интересов}

Авторы заявляют, что у них нет конфликта интересов.

\section{Список литературы}

[1] Bityurin V., Klimov A., Leonov S., Pashina A., Skvortsov V., Cain T. 1999 AIAA-99-4856 November 1999.

[2] Klimov A., Leonov S., Pashina A., Skvortsov V., Cain T., Timofeev B. AIAA-99-4856 November 1999.

[3] Leonov S., Bityurin B., Kolesnichenko Yu. AIAA 2001-0493 January 2001.

[4] Leonov S., Nebolsin V., Shilov V., Timofeev B., Kozlov A. 1999 Perspectives of MHD and Plasma Technologies in Aerospace Applications, Moscow. March 1999.

[5] Ardelyan N.V., Bychkov V.L., Kosmachevskii K.V. // IEEE Trans. Plasma Sci. 2014. Vol. 42. N 12. P. 3901-3905.

[6] Грачев Л.П., Есаков И.И., Ходатаев К.В. // ЖТФ. 1985. T. 55. C. $389-391$.

[7] Van Wie D., Risha D.J., Suchomel C.F. AIAA-20041357. 42-nd AIAA Aerospace Sciences Meeting \& Exhibit. 5-8 January 2002. Reno. NV. P. 1-10.

[8] Mashek I.Ch., Anisimov Yu.I., Lashkov V.A., Kolesnichenko Yu.F. AIAA 2005-0790. 43-rd AIAA Aerospace Sciences Meeting \& Exhibit. 10-13 January 2005. Reno. NV.

[9] Esakov I.I., Grachev L.P., Khodataev K.V. Proc. Supplement of 3-rd Weakly Ionized Gases Workshop. Waterside Marriott Hotel, Norfolk, Virginia, USA, 1-5 November, 1999, P. 99 4821.

[10] Грачев Л.П., Есаков И.И., Ходатаев К.В. // ЖТФ. 1985. T. 55. C. $972-975$.

[11] Kolesnichenko Yu.F. 2000 2nd Workshop on MagnetoPlasma-Aerodynamics in Aerospace Applications. Moscow, April 2000.

[12] Kolesnichenko Yu.F., Khmara D.V. 2000 2nd Workshop on Magneto-Plasma-Aerodynamics in Aerospace Applications. Moscow, April 2000.

[13] Kolesnichenko Yu.F., Brovkin V.G., Leonov S.B., Krylov A.A., Lashkov V.A., Mashek I.Ch., Gorynya A.A., Ryvkin M.I. AIAA-2001-0345 January 2001.

[14] Kolesnichenko Yu.F., Brovkin V.G., Azarova O.A., Gruditsky V.G., Lashkov V.A., Mashek I.Ch. 1999 Perspectives of MHD and Plasma Technologies in Aerospace Applications. Moscow, March 1999.

[15] Kolesnichenko Yu.F., Brovkin V.G., Azarova O.A., Gruditsky V.G., Lashkov V.A., Mashek I.Ch. AIAA-2003-0361 January 2003.

[16] Kolesnichenko Yu.F., Brovkin V.G., Khmara D.V., Lashkov V.A., Mashek I.Ch., Ryvkin M.I. 2004 4th Int. Workshop on Thermochemical and Plasma Processes in Aerodynamics St. Petersburg. July 2004.

[17] Soloviev V., Krivtsov V., Konchakov A., Malmuth N. AIAA2001-2727 June 2001.

[18] Soloviev V.R., Krivstov V.M., Konchakov A.M., Malmuth N.D. // AIAA J. 2003. Vol. 41. P. 2903-2909.

[19] Wilkerson J.T., Van Wie D.M., Cybyk B.Z. AIAA-2003-0526 January 2003.

[20] Грачев Л.П., Есаков И.И., Ходатаев К.В. // Матер. Междунар. симпозиума „Термохимические и плазменные процессы в аэродинамике“, СПб. 15-19 июля 2002, Холдинговая компания „Ленинец“ при участии НИПГС, EOARD, AFOSR. C. 113-119.

[21] Esakov I.I., Grachev L.P., Khodataev K.V. // Conf. Plasmas for Stealth and for Flow and Combustion Control. A joint initiative of ONERA and CNRS/SPI March 31, April 1, 2003 Institute Curie Rue d'Ulm, 75005 Paris. 
[22] Shibkov V.M., Chernikov A.V., Chernikov V.A., Ershov A.P., Shibkova L.V., Timofeev I.B., Vinogradov V.A., Voskanyan A.V. 2nd Workshop on Magneto-Plasma-Aerodynamics in Aerospace Applications. Moscow, April 2000.

[23] Shibkov V.M., Chernikov V.A., Ershov A.P., Dvinin S.A., Raffoul Ch.N., Shibkova L.V., Timofeev I.B., Van Wie D.M., Vinogradov V.A., Voskanyan A.V. AIAA-2001-3087 June 2001.

[24] Leonov S., Bityurin V., Savischenko N., Yuriev A., Gromov V. AIAA-2001-0640 January 2001.

[25] Константиновский Р.С., Шибков В.М., Шибкова Л.В. // Кинетика и катализ. 2005. Т. 46. № 6. С. 821-834. 